\title{
MÁS ACERCA DEL SISTEMA EDUCACIONAL Y LA UNIVERSIDAD DE CHILE*
}

\author{
Enrique Barros \\ Universidad de Chile \\ Arturo Fontaine \\ Universidad de Chile
}

\begin{abstract}
Resumen: En un país democrático el sistema educacional persigue una pluralidad de fines, no siempre fácilmente conciliables e irreductibles a uno sólo, tales como cobertura, calidad académica, equidad, diversidad e integración. Este ensayo aborda las distintas tensiones entre estos fines al interior del sistema educacional chileno, las dificultades de encontrar un balance entre éstos y plantea la necesidad de evaluar los diferentes instrumentos en función de ello. En este marco, se plantea la cuestión de la razón de ser de la Universidad de Chile. Los autores afirman que esta universidad, desde su origen, se dedica a la investigación y la docencia de exce-
\end{abstract}

Enrique Barros. Abogado, profesor de la Facultad de Derecho de la Universidad de Chile y miembro del Consejo Directivo del Centro de Estudios Públicos (ebarros@blc.cl).

Arturo FontainE. Profesor del Departamento de Filosofía de la Facultad de Filosofía y Humanidades de la Universidad de Chile y director del Centro de Estudios Públicos (afontaine@cepchile.cl).

* Versión ampliada del artículo que apareció en El Mercurio de Santiago el 11 de diciembre de 2011. 
lencia y no está comprometida con una determinada manera de entender el bien en la vida humana. Por cierto que hay universidades privadas inspiradas en este mismo principio de pluralismo, pero podrían cambiar mañana su naturaleza u orientación. No así la Universidad de Chile. Por otra parte - sostienen los autores - la Universidad de Chile debe revisar su sistema de gobierno y redefinir así, como universidad estatal, su vinculación con la sociedad y el sistema político.

Palabras clave: educación chilena, diversidad, integración, pluralismo, financiamiento compartido, universidad, Universidad de Chile, gobierno universitario.

Recibido y aceptado: diciembre 2011.

\section{MORE ABOUT THE EDUCATIONAL SYSTEM AND THE UNIVERSITY OF CHILE}

Abstract: In a democratic country the educational system seeks a variety of ends which cannot always be easily conciliated and reduced to a single one, such as coverage, academic excellence, equitableness, diversity and integration. This essay refers to the tensions among those ends within the Chilean educational system, the difficulties of striking a balance among them, and it deals about the need to assess the different instruments to achieve them. Within this framework, the question about the raison d' être of the University of Chile is addressed. The authors contend that since its inception this university has been a research and teaching institution, and it is not committed to a particular form of the good in human life. Of course there are private universities which are inspired by the same principle of pluralism, but they may change tomorrow their nature and orientation. This is not the case of the University of Chile. On the other side - the authors say- the University of Chile must re-examine its system of government and, as a State university, it must redefine its bonds to the society and to the political system.

Keywords: Chilean education; diversity, integration, pluralism, joint financing arrangements, university, Universidad de Chile, university government.

Received and accepted: December 2011. 


\section{E}

sistema educacional, en un país libre y democrático, ha de perseguir una pluralidad de fines: cobertura, calidad, equidad, diversidad, integración, entre otros. Puede haber tensiones entre estos fines o bienes. Buena parte de los diálogos de sordos en materia de educación se explican por ignorar que no todo se puede lograr a la vez y en el mismo lugar.

La cobertura universitaria es un bien muy aplaudido. Pero la palabra "universitaria" se ha estirado para aprovechar su prestigio. Por eso, es tan equívoco hablar sin calificaciones de una expansión de la educación universitaria. Se está hablando de realidades muy distintas. No es necesario ser románticos para saber, bajo cualquier parámetro, qué es una buena universidad. Es la que se dedica en un cierto nivel de excelencia a la investigación y a la docencia en una pluralidad de disciplinas científicas, humanistas y profesionales.

\section{La gratuidad espiritual y la excelencia académica en la universidad}

La calidad en la educación tiene dos dimensiones: la calidad académica; y la formación de hábitos y la educación del carácter. La calidad académica importa, porque contribuye a que haya focos que potencien nuestra sensibilidad, inteligencia y conocimiento. Como país, nos debe importar que haya instituciones que iluminen el camino. La universidad en su sentido clásico es una institución de excelencia que está en las fronteras del conocimiento y de las humanidades. Y es bueno para la sociedad — no sólo para ellos mismos — que haya buenos médicos o matemáticos.

Los requisitos que exigen las mejores universidades, en todo el mundo, para seleccionar a sus alumnos no deben ser interpretados sólo como un premio al mérito individual. Ser admitido en la Católica o en la de Chile, por ejemplo, depende de notas escolares y del rendimiento en pruebas de admisión, que mucho deben al talento innato, a la cultura del hogar, a los hábitos de conducta y de pensamiento que haya entregado la escuela.

En nuestra mirada a veces puramente cuantitativa de la educación superior, se suele ignorar que la dedicación a la ciencia está caracterizada por una cierta gratuidad. La ciencia no persigue otro fin prima- 
rio que comprender el mundo en que nacimos y moriremos, desentrañar sus relaciones.

"Maravillarse ante lo desconocido, y no la expectativa de beneficio que se pueda lograr gracias a los descubrimientos, es el primer principio que lleva a la humanidad al estudio de la filosofía, de esa ciencia que pretende revelar las ocultas conexiones que unen las diferentes apariencias de la naturaleza".

El improbable autor de estas ideas es Adam Smith, el gran pensador de una economía movilizada por los intercambios en un mercado abierto y sin posiciones de poder. Aunque dedicó parte de su vida a entender como operaban los intercambios económicos, le resultaba natural que la ciencia no fuera concebida como una mercancía. Smith llegó hasta los límites de su disciplina porque tenía claro que sus descubrimientos no perseguían otro fin que descubrir las complejas imbricaciones de la sociedad en que vivimos. Y por cierto que no podía prever las profundas consecuencias que produciría su forma de pensar (incluso en la biología).

Ningún país que se piense a sí mismo en serio puede renunciar a universidades de primer nivel, capaces de pensar nuevas rutas.

\section{Los fines del sistema educativo son diversos y concurrentes}

La equidad puede concebirse de diversas maneras. Un buen punto de partida es propender a que la calidad sea menos dependiente de la cuna. Si el talento natural se distribuye de manera semejante en los diversos estratos socioeconómicos, es injusto que quienes no tienen la suerte de tener padres educados o con dinero $-\mathrm{y}$ ambas cosas suelen ir juntas - no puedan ir progresivamente acortando las desventajas de origen.

A la equidad se opone una desigual calidad de la educación, que simplemente refleje y perpetúe las diferencias culturales de los hogares. Alguien podría querer incluso prohibir las escuelas y universidades privadas en aras del igualitarismo, por ejemplo. Pero este propósito es a costa de la diversidad y también de la calidad.

La diversidad, a su vez, reconoce en el plano institucional el pluralismo en lo religioso, filosófico, político o estético. De hecho, muchas de las mejores universidades privadas están orientadas por conceptos 
fuertes en algunos de esos sentidos. Lo mismo ocurre, y de manera más acentuada, en la educación escolar. En términos kantianos, las distintas instituciones promueven diversas doctrinas del bien o de la felicidad, en la medida que "la moral no es una doctrina que nos enseña cómo hacernos felices, sino cómo hacernos dignos de la felicidad". A la diversidad se opone la uniformidad educacional.

Si prima la tolerancia, el encuentro e interacción de personas heterogéneas, en la universidad o en la vida laboral, la experiencia de vida es más positiva, iluminadora y fértil. La integración apunta a que se eduquen juntos en el mismo establecimiento jóvenes de diversos estratos sociales y de distintas pertenencias culturales, religiosas y políticas. A la integración se opone la segregación.

El sistema escolar y el universitario pueden lograr estos bienes de manera más o menos equilibrada. Por ejemplo, en Chile parece haber bastante diversidad, pero mucha desigualdad en calidad; bastante cobertura, pero mucha segregación.

Un sistema puede ser muy desigual en resultados académicos y, además, segregado. Es lo que ocurre en el sistema escolar chileno. En buena medida, porque la sociedad es muy desigual y la ciudad misma está muy segregada. Pero también puede suceder que el sistema sea bastante integrado socialmente y, sin embargo, desigual. Esto parece ocurrir en Francia, por ejemplo, donde en promedio los resultados escolares dependen aún más del hogar que en Chile (PISA, 2009). Sistemas estatales y gratuitos no aseguran per se la integración ni la equidad.

El financiamiento compartido, por ejemplo, introducido como opción en el sistema escolar en 1993, siendo Ministro de Educación don Jorge Arrate, apunta a mejorar la calidad con aportes de los padres. También contribuye a la diversidad de proyectos educacionales. Cumple fines valiosos, aunque no esté dirigido a aumentar la integración.

Algunos incentivos pueden promover la integración. Por ejemplo, premiar económicamente a las universidades y a los colegios que acepten alumnos vulnerables. La subvención preferencial podría empujar las cosas en esa dirección (siempre que los padres sepan cuando la tienen). Sin embargo, si ese premio pasa de cierto umbral, resulta perjudicada la calidad.

Algunas críticas a nuestro sistema educacional —al financiamiento compartido y a la segregación - están en la línea de Bruce Ackerman (Social Justice in a Liberal State). Ackerman ve una fuerte 
tensión entre el interés de los padres por que la educación externa refuerce la educación de la familia, y el de la sociedad por que los jóvenes definan sus propios ideales, reconociendo su libertad moral. Los padres, por así decirlo, quieren formar herederos y el estado, ciudadanos.

El supuesto es que sólo se puede educar ciudadanos con la actitud crítica característica de una sociedad democrática si los jóvenes conviven desde temprano con otros de distintas proveniencias sociales y económicas, con diversas visiones de mundo. Ello supondría prohibir tanto la educación particular pagada, como la particular subvencionada. Siguiendo la misma lógica no debiera haber universidades privadas. Se trata de una tesis que da importancia superior a la integración como fin del sistema educacional. Aunque ello signifique sacrificar otros bienes valiosos.

No ha sido el enfoque que ha predominado históricamente en Chile. El estado empezó a financiar escuelas y universidades privadas mucho antes de que hubiera nacido Milton Friedman, el gran promotor de los vouchers (J. L. Ossa, Estudios Públicos 106, 2007). Las subvenciones fueron introducidas para multiplicar los recursos en educación, pero también para dar cuenta del pluralismo, de la contienda entre católicos y laicos. Y para apoyar los esfuerzos educacionales de los franciscanos en la Araucanía, y de los alemanes en el Sur; y también para consolidar la educación femenina, por ejemplo.

La cuestión no es simple, porque no es obvia la elección entre diversidad e integración; ni entre calidad y cobertura. Acostumbramos poner acento en los instrumentos, pero quizás atendemos poco acerca a qué bienes o fines queremos conseguir y de qué manera un bien valioso puede resultar ignorado si la discusión se ideologiza. La regulación y orientación del sistema educacional debe hacerse cargo de una pluralidad de fines, buscando un balance adecuado.

Por ejemplo, todo indica que la explosiva expansión de la matrícula universitaria es un juego de máscaras, en que los más desprotegidos hacen como que estudian lo que no están en condiciones de aprender y algunas instituciones hacen como si fueran universidades de verdad. Atendidas las limitaciones de capital cultural de muchas familias chilenas y los actuales estándares de la enseñanza escolar, lo más razonable para dar un salto cualitativo, evitando esa ficción, es una educación técnica de calidad; no una educación que se dice universitaria, pero que terminará produciendo frustraciones. 
La legislación de 1981 promovió que toda carrera se convirtiera en licenciatura; de cinco años, además. En razón de incentivos erróneos, recién ahora se están igualando los estudiantes de profesiones técnicas con la matrícula universitaria. Y universidades privadas de calidad, que persiguen fines no lucrativos, consistentes con la misión de una genuina universidad, son metidas en el mismo cambucho que las que ofrecen falsas ilusiones.

\section{¿Por qué la Universidad de Chile?}

Dado este marco, ¿por qué es necesaria la Universidad de Chile? Cuando el Estado la fundó a comienzos de la República, quiso asegurar la existencia de al menos una universidad nacional de excelencia, consagrada a la investigación y docencia. El Estado inspirado "por las más sanas y liberales ideas, ha encargado a la universidad, no sólo la enseñanza, sino el cultivo de la literatura y las ciencias...; que contribuyese al aumento y desarrollo de los conocimientos científicos, que no fuese sólo un instrumento pasivo, destinado exclusivamente a la transmisión de los conocimientos ya adquiridos..." (Andrés Bello, Discurso en el aniversario de la Universidad de Chile, 1848).

A diferencia de otras instituciones de la enseñanza superior que sólo se dedican a la docencia, la Universidad de Chile, desde su fundación, ha aspirado a ser una institución selectiva, consagrada a la enseñanza que se sustenta en la investigación. Eso supone hoy equipos académicos estables de alto nivel, escogidos por su mérito.

$\mathrm{La}$ experiencia internacional muestra que instituciones de esta naturaleza, si son privadas, no tienen fines de lucro y cuentan con un patrimonio que les ha sido donado por particulares; y si son instituciones estatales, su financiamiento proviene principalmente (pero no exclusivamente) del Estado. A unas y otras, el Estado hace aportes vía becas y recursos para la investigación.

Al destinar recursos a esos departamentos académicos, el país no sólo fortalece la investigación científica y humanística y, en general, las profesiones que se nutren de tales disciplinas matrices. Muy especialmente, éstas contribuyen a mejorar la educación en el país, porque sólo los profesores que aman y dominan su disciplina pueden enseñarla fructíferamente. Por eso, el desarrollo de las disciplinas básicas a nivel 
universitario es el mejor camino para generalizar el expandir la calidad de la educación escolar y combatir la desigualdad.

La Universidad de Chile no está comprometida con una determinada manera de entender el bien en la vida humana. Por cierto que hay universidades privadas inspiradas en el mismo principio, pero podrían cambiar mañana su naturaleza u orientación. No así la Universidad de Chile. El Estado y la sociedad chilena tienen el deber de sustentar este proyecto universitario nacional.

Se trata de un espacio donde se puedan analizar y poner en cuestión las más diversas formas de vida; donde la diversidad no sólo es teoría, sino presencia real. En la Universidad de Chile el pluralismo se vive cotidianamente con toda naturalidad. Un profesor de la universidad puede tener las convicciones más diversas y, sin embargo, las transmite con completa libertad. La interacción intelectual y humana que eso permite es una oportunidad muy valiosa para sus estudiantes y profesores. En especial para sus muchos alumnos que estudiaron en colegios homogéneos. La Universidad de Chile representa los ideales de meritocracia y diversidad, que dan forma a una experiencia formativa que abre horizontes y amplía la visión de la vida; y ello es muy valioso en una sociedad tan segmentada como la chilena.

Pero para que haya integración no basta la mera yuxtaposición de estudiantes de diversos estratos socioeconómicos, y vertientes culturales, políticas y religiosas. Se requiere que en su interior primen prácticas enraizadas de comunicación mutua, de respeto por el otro, de análisis racional, de discusión informada y de espíritu de unidad en la pluralidad.

Muchos pueden temer que la Universidad de Chile tome el camino decadente de algunas universidades públicas hispanoamericanas. Por eso, la universidad debe cuidar que desde ella misma surjan propuestas de buen gobierno, que la acerquen a las mejores universidades públicas. El corporativismo, que subyace a su actual forma de gobierno, favorece la composición de los intereses particulares en un archipiélago de facultades e institutos (en incluso dentro de éstos). Además, dificulta las conexiones vitales de la universidad con la sociedad en que interactúa. Las universidades públicas más exitosas se han alejado de ese modelo, que ha resultado ruinoso en tantos lugares de nuestro continente. Que la Universidad de Chile sea pública exige una mayor participación de toda la sociedad chilena en el cuidado y cumplimiento de sus fines. 
La educación escolar tiene en Chile más de socialización que de autocreación. En la Universidad de Chile el énfasis debe estar puesto un poco más en el examen y en la creación del yo. Para ello es necesario un contacto habitual con el pensamiento teórico, las obras de arte y las tradiciones intelectuales que impregnan la manera como sentimos, imaginamos y pensamos. Como contrapartida, se requiere un ambiente de trabajo estimulante y una adecuada retribución para sus académicos. Todo ello supone revisar su régimen de gobierno y, más profundamente, sus relaciones con la sociedad chilena.

Un estudio comparado realizado por uno de nosotros muestra que las más exitosas universidades públicas en tres continentes son gobernadas por un consejo directivo compuesto por académicos y por personalidades externas a la universidad, que tienen experiencia en gestión pública o empresarial. A ese órgano de composición plural corresponde definir la estrategia, para que la universidad cumpla su misión académica de excelencia, y designar y remover al rector. El gobierno universitario simplemente debe facilitar que esa peculiar forma de vida - la universitaria - florezca en un ambiente de creciente excelencia y libertad.

Las buenas universidades son comunidades de estudiantes y profesores dedicadas a la preparación profesional, pero también a la conversación intelectual libre, rigurosa e indagatoria. Son comunidades dedicadas a la exploración por la belleza y asombro de la exploración misma. En esas conversaciones, estudios y exploraciones se teje parte importante del futuro de nuestro país; como el de toda sociedad que quiera ser mejor. 